\title{
DELTA WINGS WITH SHOCK-FREE CROSS FLOW*
}

BY

\author{
S. S. SRITHARAN \\ Institute for Computer Applications in Science and Engineering
}

\begin{abstract}
It has been realized recently that in order to have a high level of maneuverability, supersonic delta wings should have a cross flow that is free of embedded shock waves. The conical cross flow sonic surface differs from that of plane transonic flow in many aspects. Well-known properties such as the monotone law are not true for conical cross flow sonic surfaces. Using a local analysis of the cross flow sonic line, relevant conditions for smooth cross flow are obtained. Using a technique to construct artificially a smooth sonic surface and an efficient numerical method to calculate the flow field, one obtains cones with smooth cross flow.
\end{abstract}

Introduction. It is well known that the most suitable structure for a slender wing airplane has leading edge separation [1]. The resulting vortices are highly stable, and while contributing to the induced drag, also increase the lift because of the low pressure they induce. These vortices usually increase the lift-to-drag ratio for a delta wing. However, the modern supercruiser concept demands efficient supersonic cruise and high-level supersonic, as well as transonic, maneuverability [2]. In order to obtain this level of performance, the wings have to be relatively thick and should have transonic leading edges and attached flow [3]. A delta wing with transonic leading edge will, in general, have embedded cross flow shocks. To obtain attached flow, this configuration should have a cross flow that is free of embedded shocks or should contain only weak shock waves. Present study is tailored to find such configurations but is limited to conical wings and irrotational flow. Using a novel technique to construct smooth embedded sonic surfaces, shock-free cross flow is constructed for the first time for conical wings. We have also provided, based on a local analysis, the necessary geometric condition that should be satisfied by a configuration in order for a shock-free cross flow to exist.

Irrotational conical flows. Let $\left\{\xi^{\alpha}\right\}$ be the surface coordinates of a unit sphere centered at the apex of the cone and $g_{\alpha \beta}$ be the corresponding metric tensor. Let $V^{\alpha}$ be the tangent

\footnotetext{
* Received February 27, 1984. This research was supported by the National Acronautics and Space Administration under NASA Contract No. NAS1-17070 while the author was in residence at ICASE. NASA Langley Research Center, Hampton, Virginia.
} 
velocity field (Fig. 1) generated on the sphere by the mainstream flow. Irrotational assumption implies

$$
V^{\alpha}=g^{\alpha \beta} \partial F / \partial \xi^{\beta}, \quad \alpha, \beta=1,2,
$$

where $F$ is the conical potential. The total velocity $q$ is given by

$$
q^{2}=V^{\alpha} V_{\alpha}+F^{2} \text {. }
$$

The continuity equation is [4],

$$
\left(\rho V^{\alpha}\right)_{\| \alpha}+2 \rho F=0
$$

where $\|$ denotes surface covariant differentiation and $\rho$ is the gas density and is given by the Bernoulli equation,

$$
\rho^{\gamma-1}=1+\frac{\gamma-1}{2} M_{\infty}^{2}\left(1-q^{2}\right) .
$$

Here $M_{\infty}$ is the free stream Mach number and $\gamma$ is the ratio of specific heats. Combining Eq. (1) and (2), we obtain the quasilinear form of the governing equation,

$$
\left(g^{\alpha \beta}-\frac{V^{\alpha} V^{\beta}}{a^{2}}\right) V_{\alpha \| \beta}+\left(2-M_{c}^{2}\right) F=0,
$$

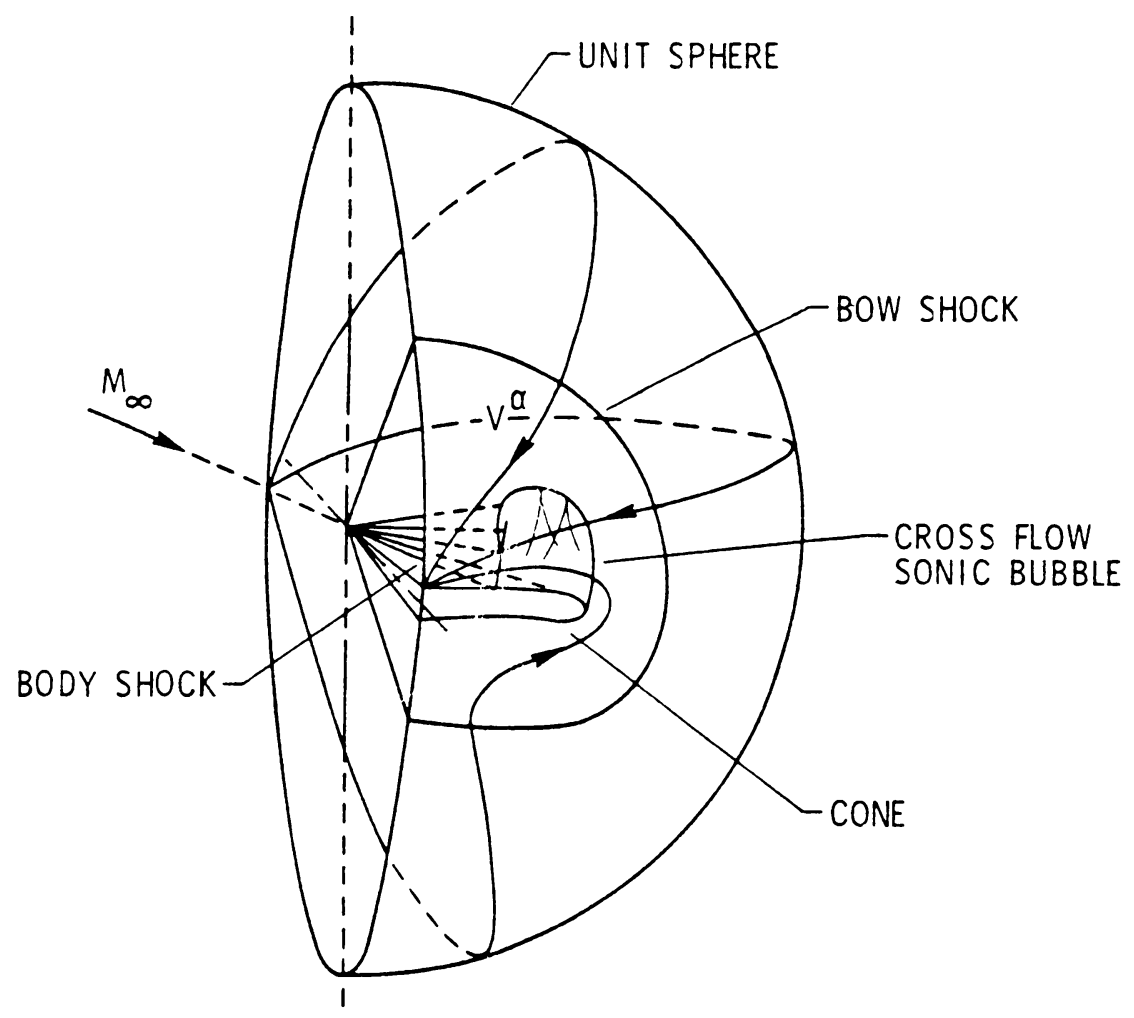

FIG. 1. The classical tangent field representation of conical flows on unit sphere. 
where $a$ is the speed of sound and $M_{c}$ is the cross flow Mach number $\left(M_{c}=q_{c} / a\right)$. The Eq. (3) changes from elliptic to hyperbolic type when $M_{c}$ increases through unity. The above behavior is similar to plane transonic flows and therefore can be utilized to develop an extremely efficient numerical method to calculate supersonic flows past arbitrary conical shapes at incidence [5].

Cross flow sonic surfaces. Sonic bubbles that appear in conical cross flows differ from those of transonic flows in many aspects. In transonic flow the flow properties attain uniform state on the sonic line, whereas in conical flows the appearance of the radial velocity term in the Bernoulli equation causes the flow properties to vary along the sonic line. Plane transonic flow is governed by a homogeneous set of partial differential equations, and therefore, it is possible to obtain a linear problem through a Legendre transformation. This property also gives us the well-known Nikolskii-Taganov monotone rule [6]: if an observer moves along the sonic line, keeping the subsonic zone always to his left, then the stream vector will rotate in the clockwise direction. However, in conical flows, due to the inhomogeneous terms in the governing equations, it is not possible to obtain linear equations using a hodograph transformation, and also it is difficult to say anything definite about the streamline slope. Some properties of conical sonic surfaces have been worked out by Salas [7], and with the aid of some of his results, we will study some relevant aspects for shock-free flows.

First we note an interesting behavior of the pressure at the point where a cross flow streamline exists the hyperbolic zone. Consider the Bernoulli equation

$$
\frac{1}{2}\left(q_{c}^{2}+F^{2}\right)+\frac{\gamma}{\gamma-1} \frac{p}{\rho}=\text { constant }
$$

taking the derivative in the $s$ direction and substituting the adiabatic relation

$$
p=\frac{1}{\gamma M_{\infty}^{2}} p^{\gamma},
$$

We get

$$
\frac{\partial p}{\partial s}=-p q_{c}\left(\frac{\partial q_{c}}{\partial s}+F\right) .
$$

Now, from the definition of the cross flow Mach number

$$
\frac{\partial M_{c}}{\partial s}=\frac{1}{a}\left[\frac{\partial q_{c}}{\partial s}-M_{c} \frac{\partial a}{\partial s}\right] .
$$

Using this and the energy equations, we obtain the relations at the sonic surface for the streamwise pressure gradient, namely

$$
\frac{\partial p}{\partial s}=-\left(\frac{2}{\gamma+1}\right) \rho q_{c}\left[q_{c} \frac{\partial M_{c}}{\partial s}+F\right] .
$$

From this we see that since $F$ is always positive, the pressure always decreases when the streamline enters the hyperbolic zone, which is similar to transonic flows. However, when it exists the hyerbolic zone, the pressure could either increase or decrease. This is in contrast to what happens in transonic flows, where the pressure always increases as the 
streamline exits the sonic surface because the radial velocity term $F$ does not appear in the equation for $\partial p / \partial s$. This possibility leads us to believe that a shock-free situation is more likely in conical flows. We recall here that in transonic flows the shock-free situation has been proved to be mathematically isolated [8]. (It has been established experimentally [9], [10] that the neighboring flows have only weak shock waves.) A similar perturbation theory for shock-free conical flows does not yet exist and shock-free solutions have not previously been shown to exist.

Next, we will observe a unique feature of the cross flow sonic line. We will first obtain a description for the sonic line by expanding the cross flow velocity and the speed of sound and equating them. Thus we write

$$
\begin{gathered}
a=a^{*}+\left(\frac{\partial a}{\partial s}\right) * d s+\left(\frac{\partial a}{\partial n}\right) * d n+\ldots \\
q_{c}=a^{*}+\left(\frac{\partial q_{c}}{\partial s}\right) * d s+\left(\frac{\partial q_{c}}{\partial n}\right) * d n+\ldots
\end{gathered}
$$

and also, from the energy equation,

$$
\left(\frac{\partial a}{\partial n}\right) *=-\left(\frac{\gamma-1}{2}\right)\left(\frac{\partial q_{c}}{\partial n}\right) *
$$

Thus, the equation of the line $q_{c}=a$ is

$$
\left(\frac{d n}{d s}\right)_{\text {sonic line }}=-\left(\frac{2}{\gamma+1}\right) a^{*} \frac{\left(\partial M_{c} / \partial s\right)^{*}}{\left(\partial q_{c} / \partial n\right)^{*}} .
$$

This relation indicates that the angle at which the cross flow sonic line meets the body is determined by the sign of $\left(\partial q_{c} / \partial n\right)^{*}$.

This means that if this quantity is negative, the sonic line could meet the body at an obtuse angle. Salas incorrectly predicts this possibility for a circular cone and then uses an argument based on pressure to speculate on the possibility of a shock wave. However, one could rule out the existence of smooth flow with this kind of a bubble, using the following argument.

At the sonic line, the characteristics are normal to the streamlines, and therefore, from Fig. 2, it is clear that for a sonic line of this kind, characteristics of the same family will intersect. Thus, if through the above analysis, we arrive at a sonic line of this type, then for this cone a shock-free cross flow is impossible. In fact, one may work out rules to identify conical shapes for which shock-free cross flow is impossible. For convenience, we will work in the stereographically projected plane. If one chooses a Cartesian system $(x, y)$ in this plane, then the metric tensor will be

$$
g_{\alpha \beta}=\left[\begin{array}{cc}
J^{2} & 0 \\
0 & J^{2}
\end{array}\right],
$$

where

$$
J=\frac{2}{1+x^{2}+y^{2}} .
$$


If $\hat{\alpha}$ denotes the inclination of the streamline with

$$
d y / d x=\tan \hat{\alpha},
$$

then the irrotationality condition becomes

$$
\frac{\partial q_{c}}{\partial n}=q_{c}\left[\frac{\partial \hat{\alpha}}{\partial s}-\frac{1}{J} \frac{\partial J}{\partial n}\right] .
$$

We have already noted that when

$$
\partial q_{c} / \partial n<0
$$

at the body, shock-free cross flow on a general cone is impossible, and hence cones with

$$
\frac{1}{R}-\frac{\partial J}{\partial n}<0
$$

shock-free cross flow is impossible.

We note that

$$
\begin{aligned}
\frac{\partial}{\partial s} & =\frac{1}{J} \cos \hat{\alpha} \frac{\partial}{\partial x}+\frac{1}{J} \sin \hat{\alpha} \frac{\partial}{\partial y} \\
\frac{\partial}{\partial n} & =-\frac{1}{J} \sin \hat{\alpha} \frac{\partial}{\partial x}+\frac{1}{J} \cos \hat{\alpha} \frac{\partial}{\partial y} \\
d s^{2} & =J^{2}\left(d x^{2}+d y^{2}\right),
\end{aligned}
$$

and $R$ is the radius of curvature of the image of the cone in the stereographically projected plane. Thus, cones that satisfy the above rule will not have a shock-free cross flow.

We will now show that this condition is not satisfied on a circular cone. Consider a circular cone of half angle $\psi_{\text {. }}$. Then the radius of the circle on the stereographically projected plane is

$$
a=\tan \frac{\psi_{c}}{2}
$$

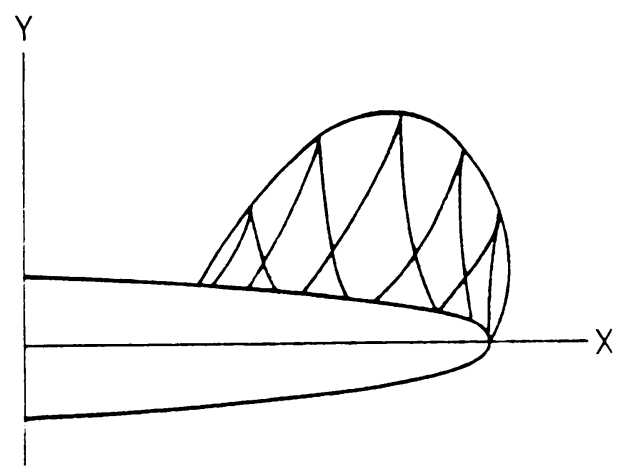

SHOCK FREE CROSSFLOW

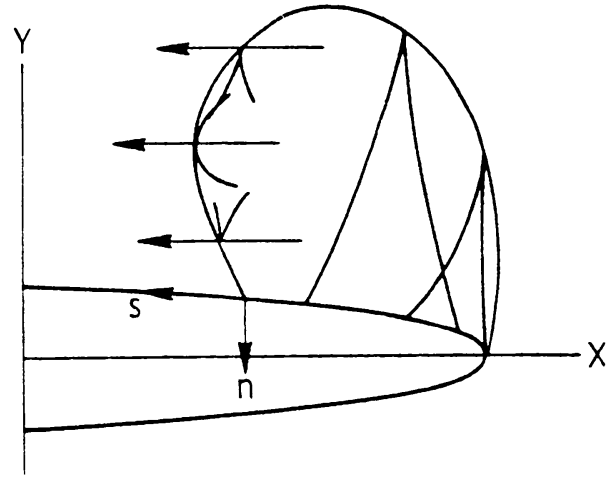

SONIC LINE MEETING THE BODY AT AN OBTUSE ANGLE

FIG. 2. Types of sonic lines that terminate in the body. 
since

$$
\psi_{c}<90^{\circ}, \quad a<1 \text {. }
$$

Thus on the body

$$
J=2 /\left(1+a^{2}\right)
$$

and

$$
\frac{\partial q_{c}}{\partial n}=\frac{q_{c}}{J}\left(\frac{1}{a}-J a\right)>0
$$

A design method to obtain conical wings with shock-free cross flow. It is possible to devise a direct approach to search for shock-free configurations. Suppose we consider a test case with shocked cross flow and change the expression for the density (equations) inside the cross flow bubble in such a way that the resulting partial differential equation is elliptic; then the elliptic-to-elliptic transition will result in a smooth sonic surface.

We may accept the solution outside this surface (which includes the bow shock wave and part of the cone) and use the flow properties on this surface to solve the Cauchy problem for the actual gas law (for the actual gas law, the governing equation is hyperbolic) to obtain the new body shape inside this surface. However, one should note that in this method there is no guarantee that a certain gas law will provide Cauchy data that will provide a smooth flow up to the body. This method is still preferable because it is direct and only part of the configuration is being modified. This method has been successfully introduced to transonic flows by Sobieczky [11]. The application of this fictitious gas method is not straightforward for conical flows for the following reasons.

In plane transonic flow, a sonic bubble is defined by the statement $q \geqslant a^{*}$, where $a^{*}$ is the speed of sound at the sonic condition and is a known constant. In conical flows, however, $a^{*}$ varies and thus is an unknown. This difficulty can be eliminated by first computing the actual speed of sound and, whenever it is less than the cross flow velocity, replacing it by the fictitious speed of sound. When we change the gas law, we must take care to preserve mass conservation at the sonic line. In conical flows, because the density varies along the sonic line, the gas law should be chosen to give continuous density across the sonic line. Let us look at the simplest way to meet these requirements. The energy equation is

$$
M_{\infty}^{2} a^{2}=\rho^{\gamma-1}=1+\frac{\gamma-1}{2} M_{\infty}^{2}\left(1-q_{c}^{2}-F^{2}\right),
$$

and at the sonic conditions

$$
M_{\infty}^{2} a_{*}^{2}=\rho_{*}^{\gamma-1}=\frac{1+([\gamma-1] / 2) M_{\infty}^{2}\left(1-F_{*}^{2}\right)}{([\gamma+1] / 2)} .
$$

Thus, if we use the gas law of the form

$$
M_{\infty}^{2} a^{2}=\rho^{\gamma-1}=\frac{1+([\gamma-1] / 2) M_{\infty}^{2}\left(1-F^{2}\right)}{([\gamma+1] / 2)},
$$


then the flow properties would be continuous across the sonic line and the resulting partial differential equation will have the form

$$
g^{\alpha \beta} \frac{\partial^{2} F}{\partial \xi^{\alpha} \partial \xi^{\beta}}+\cdots=0
$$

and be elliptic. This could be called the incompressible analogue for conical flows. In changing the gas law inside the sonic surface, one needs to make sure that the gas law near the bow shock wave is correct.

This was done by first solving the real problem to convergence, so that the bow shock and the cross flow sonic bubble were well developed, and then using this as the initial condition to solve the problem with the fictitious gas law inside the sonic surface. Inside the bubble the artificial viscosity [5] should be switched off and the iteration scheme should be specialized to the case of $a \rightarrow \infty$. We will now consider the Cauchy problem for the cross flow bubble. The domain $\Omega_{2}$ is first mapped to a rectangle, using an "onion peel" transformation as shown in Fig. 3. Since this transformation is singular at the end points, it is decided to use the covariant velocities in the spherical polar coordinate system $(\theta, \psi)$ as dependent variables.

Let

$$
U=F_{\theta} \text { and } V=F_{\psi}
$$

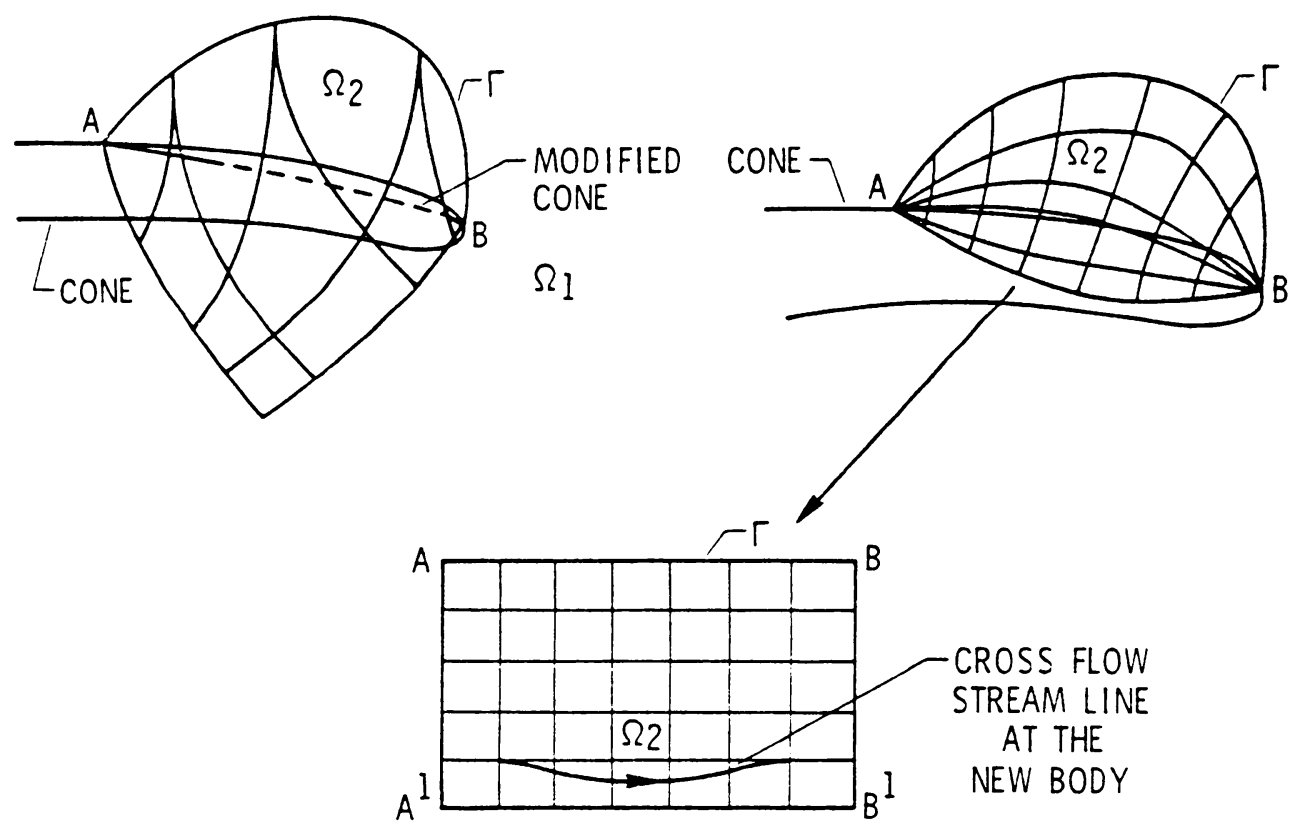

FIG. 3. 
then the governing equation is

$$
A U_{\theta}+B\left(U_{\psi}+V_{\theta}\right)+C V_{\psi}+D=0
$$

and the irrotationality gives

$$
U_{\psi}-V_{\theta}=0,
$$

where

$$
\begin{aligned}
& A=\frac{1}{\sin ^{2} \psi}-\frac{U^{2}}{a^{2} \sin ^{4} \psi}, \\
& B=-\frac{U V}{a^{2} \sin ^{2} \psi}, \\
& C=1-\frac{V^{2}}{a^{2}}
\end{aligned}
$$

and

$$
D=\left(2-M_{c}^{2}\right) F+V \cot \psi\left(1+\frac{U^{2}}{a^{2} \sin ^{2} \psi}\right) .
$$

The derivatives of covariant velocities are now transformed from $(\theta, \psi)$ to $(\zeta, \eta)$ plane. Thus we get

$$
\vec{U}_{\eta}=P \vec{U}_{\xi}+\vec{Q}
$$

Here

$$
\vec{U}=\left(\begin{array}{c}
F_{\theta} \\
F_{\psi}
\end{array}\right)
$$

The Cauchy problem is then solved by a predictor-corrector method. We write

$$
\begin{aligned}
F^{*} & =F^{0}+\Delta \eta\left(U \theta_{\eta}+V \psi_{\eta}\right)^{0}, \\
\vec{U}^{*} & =U^{0}+\Delta \eta\left(P^{0}\left(\delta_{\xi}^{-} \vec{U}^{0}\right)+\vec{Q}^{0}\right), \\
F^{+} & =\frac{1}{2}\left[F^{*}+F^{0}+\Delta \eta\left(U \theta_{\eta}+V \psi_{\eta}\right)^{*}\right], \\
\vec{U}^{+} & =\frac{1}{2}\left[\vec{U}^{*}+\vec{U}^{0}+\Delta \eta\left(P^{*}\left(\delta_{\xi}^{+} \vec{U}^{*}\right)+\vec{Q}^{*}\right)\right] .
\end{aligned}
$$

On the well-posedness of the marching problem. The marching problem would be well posed in the absence of any kind of singular points or limit lines. Conical equations do allow the cross flow streamline to meet the sonic line at right angle. However, at this point, the characteristics are tangent to the sonic line and the marching is not well posed. This situation occurs for two kinds of sonic lines, as shown in Fig. 4. Only type $(B)$ is relevant for our design. From the earlier arguments we see that for this kind of bubble, the same family of characteristics will intersect, and therefore if the fictitious gas chosen leads to a bubble of this shape, it should be discarded. 
A sonic line that does not have such a singular point on it could still lead to a limit line (as shown in Fig. 5) in the subsequent marching. If this limit line occurs above the body, then this sonic line should again be discarded.

The Jacobian's

$$
J_{G}=\theta_{\xi} \psi_{\eta}-\theta_{\eta} \psi_{\xi}
$$

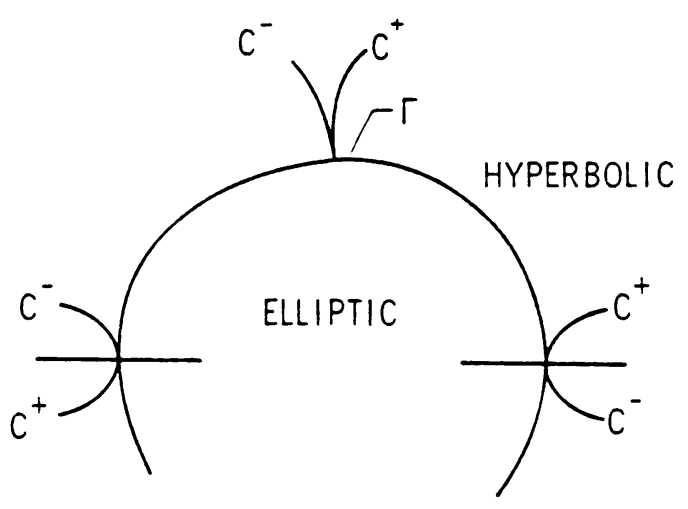

A

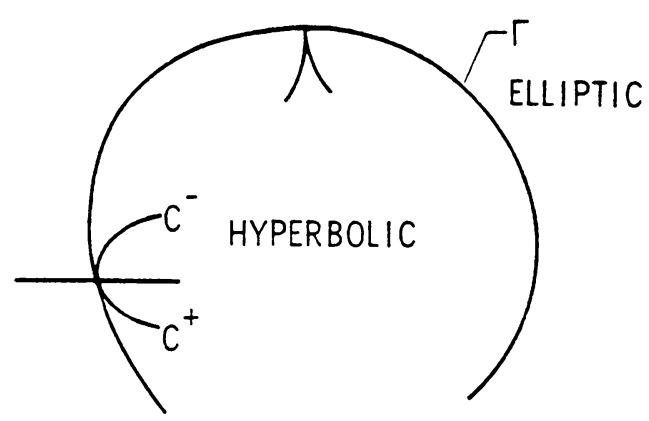

B

FIG. 4

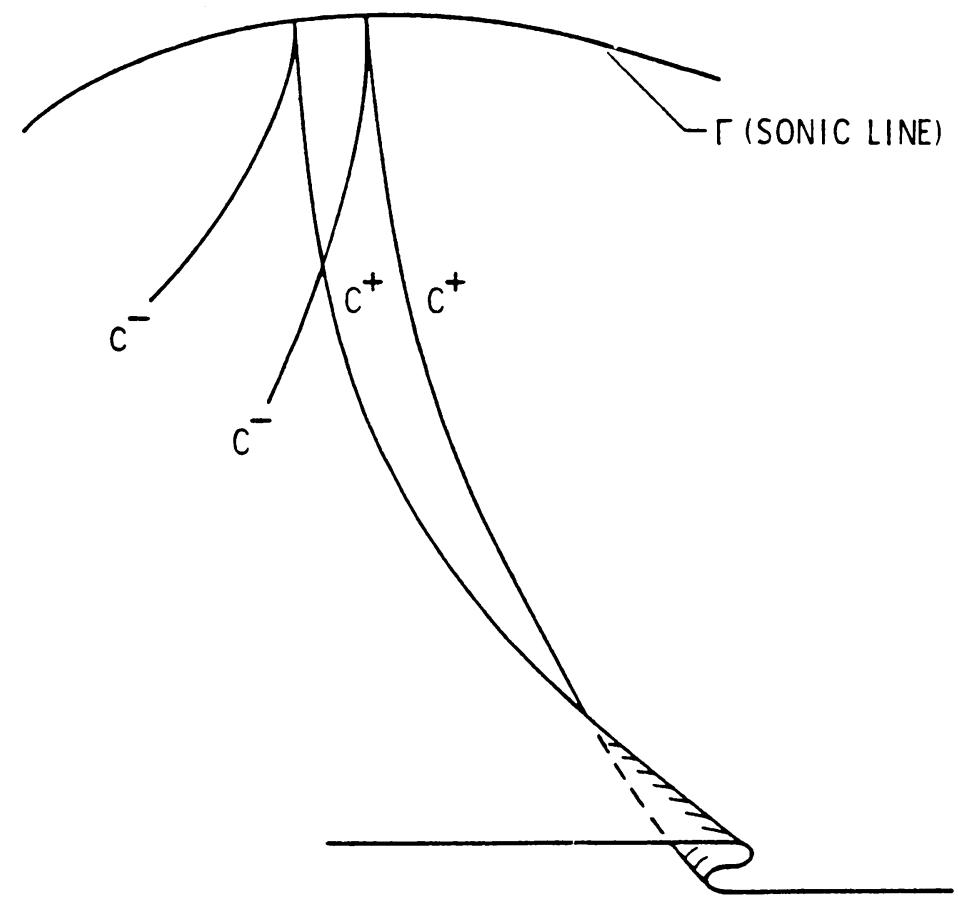

FIG. 5. Limit line. 
and

$$
J_{L}=-A \eta_{\theta}^{2}-2 B \eta_{\theta} \eta_{\psi}-C \eta_{\psi}^{2}
$$

respectively, signal grid singularities and limit lines by changing their signs.

Results. A simple test case is given to validate the method. A circular cone of $10^{\circ}$ half angle at an angle of attack of $20^{\circ}$ at Mach 2 is considered. Fig. 6 and 7, respectively, show the sonic surfaces and pressure distributions for the original as well as the modified cone. Fig. 8 shows the required surface modification of the cone. In the actual design process the fictitious gas method should be combined with a change in camber to produce the required performance.

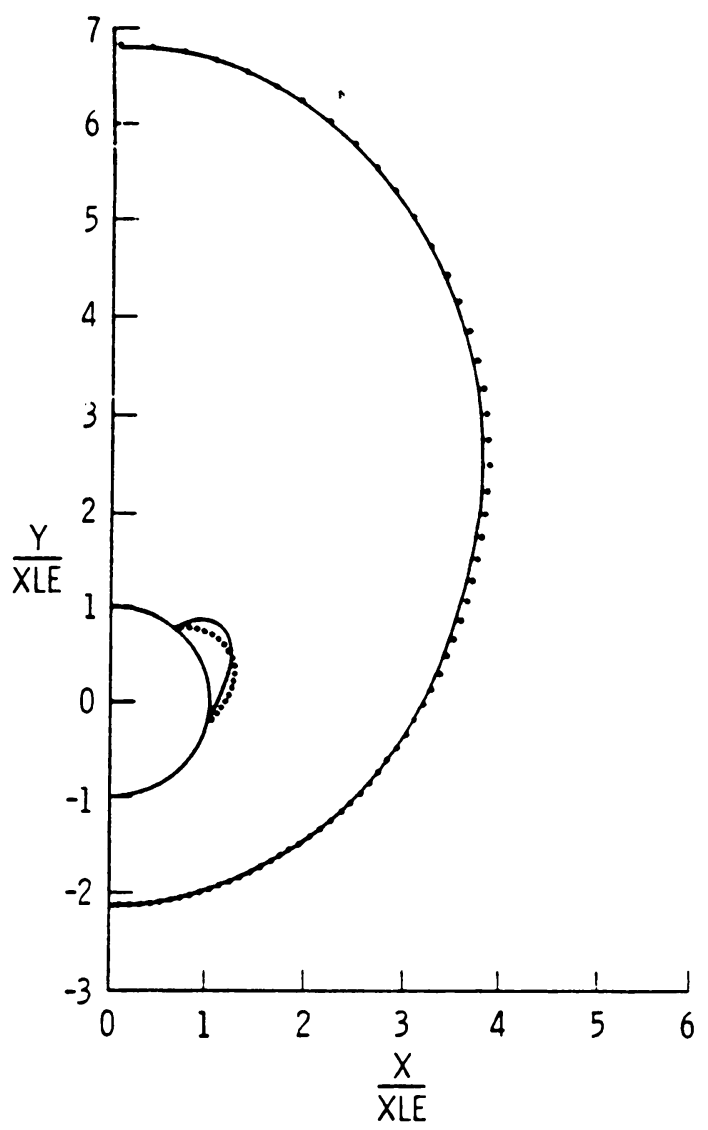

Fig. 6. Bow shock position and the cross flow sonic line for a $10^{\circ}$ circular cone at $20^{\circ}$ angle of attack at $M_{\infty}=2$. original cone, modified cone. 


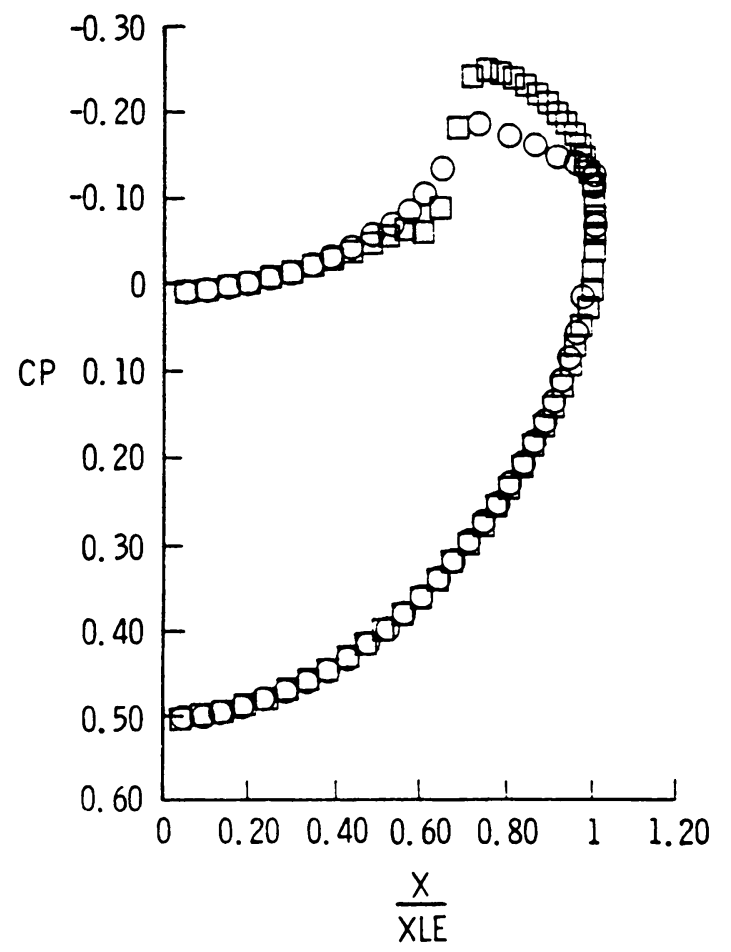

FIG. 7. Surface pressure distribution on the circular cone. $\square$ original cone, $O$ modified cone.

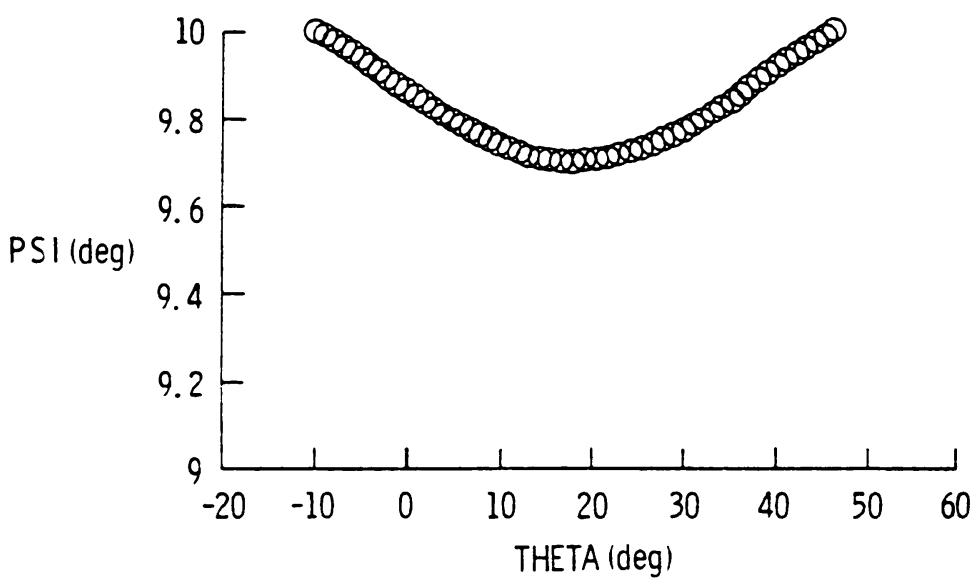

FIG. 8. Surface modification of the $10^{\circ}$ cone. Cone surface is described in the spherical polar coordinate system with theta $=$ latitude and $\mathrm{psi}=$ longitude. 


\section{REFERENCES}

[1] D. Küchemann, The aerodynamic design of aircraft, Pergamon Press, 1978

[2] $\mathrm{Ph}$. Poisson-Quinton, Slender wings for civil military aircraft, Israel Journal of Technology, 16, 97-131, (1978)

[3] W. H. Mason and D. S. Miller, Controlled supercritical cross flow on supersonic wings-An experiment validation, AIAA 13th Fluid and Plasma Dynamic Conference, Snowmass, CO, Paper No. 80-1421, July 14-16, 1980.

[4] S. S. Sritharan, Nonlinear aerodynamics of conical delta wings, Ph.D. Thesis, August 1982, Applied Mathematics, University of Arizona.

[5] S. S. Sritharan and A. R. Seebass, A finite area method for nonlinear supersonic conical flows, AIAA J.. 22. 226-233 (1984)

[6] A. A. Nikolskii and G. I. Taganov, Gas motion in a local supersonic region and conditions of potential flow breakdown, N.A.C.A. Technical Memorandum No. 1213, May 1949

[7] M. D. Salas, Flow patterns near a conical sonic line, 17th Aerospace Sciences Meeting, New Orleans, LA. January 15-17, 1979, No. 79-0341

[8] C. S. Morawetz, The mathematical approach to the sonic barrier, Bull. Amer. Math. Soc., 6, 2, 127-145 (1982)

[9] H. H. Pearcey, The aerodynamic design of section shapes for swept wings, Adv. in Aeronautical Sciences, 3. (1962) 277-320

[10] R. T. Whitcomb, Review of NASA supercritical airfoils, 9th Intl. Congress Aeronautical Sciences, Haifa, Israel, 1974

[11] H. Sobieczky, N. J. Yu, K.-Y. Fung, and A. R. Seebass, New method for designing shock-free transonic configurations, AIAA J., 17, 7, 722-728 (1979) 